\title{
Estimation for Nonlinear Dynamical Systems over Packet-Dropping Networks
}

\author{
Zhipu Jin Chih-Kai Ko and Richard M. Murray
}

\begin{abstract}
Two approaches, extended Kalman filter (EKF) and moving horizon estimation (MHE), are discussed for state estimation for nonlinear dynamical systems over packetdropping networks. For EKF, we provide sufficient conditions that guarantee a bounded EKF error covariance. For MHE, a natural scheme on organizing the finite horizon window is proposed to handle intermittent observations. A nonlinear programming software package, SNOPT, is employed in MHE and the formulation for constraints is discussed in detail. Examples and simulation results are presented.

Index Terms - State estimation, nonlinear dynamical systems, extended Kalman filter, moving horizon estimation, packetdropping networks.
\end{abstract}

\section{INTRODUCTION}

Because of the technical advances in communication and computation, networks have become ubiquitous in today's environment and the theory of networked control systems (NCSs) is an active research area [1]. Fig. 1 shows the structure of a typical NCS. Unlike traditional control theory, measurements and control signals in an NCS travel through non-ideal communication networks in which information may be delayed, re-ordered, or even dropped. In this paper we study an interesting problem in the NCS theory: state estimation for nonlinear dynamical systems over packet-drop networks.

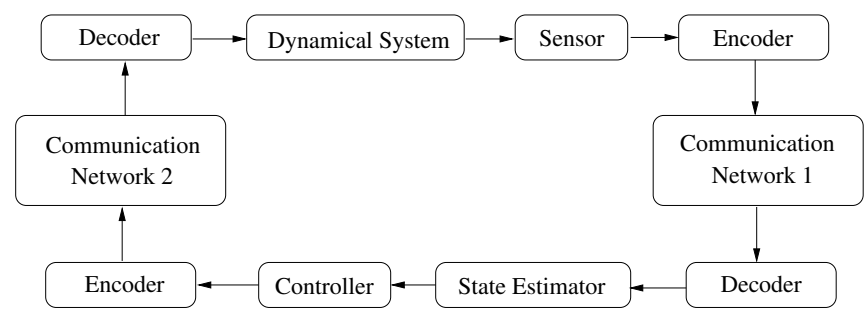

Fig. 1. Diagram of a typical networked control system.

Some exciting progress has been reported in the area of linear estimation over packet-dropping networks. Studies on filtering with intermittent observations can be traced back to [2] and [3]. Other researchers try to model the Kalman filter with missing observations as a jump linear system,

Zhipu Jin is with the Department of Mathematics, University of California, Los Angeles, USA. He also holds a position as visiting scholar in the Department of Control and Dynamical Systems, California Institute of Technology. Email: zhipu@math.ucla.edu

Chih-Kai Ko is with the Department of Electrical Engineering, California Institute of Technology, Pasadena, CA, USA. Email: cko@caltech.edu

Richard M. Murray is with Faculty of the Department of Control and Dynamical Systems, California Institute of Technology, Pasadena, CA, USA. Email: murray@cds.caltech.edu which is a stochastic hybrid system with linear dynamics and discrete Markov chains. Certain convergence criterions are given for expected estimation error covariance in [4] and [5]. More recently, Sinopoli et al. in [6] study the behavior of the Kalman filter over an i.i.d. Bernoulli packetdropping channel. They notice that there exists a critical dropping probability, (i.e. a phase transition), above which the estimation error covariance diverges. Jin et al. in [7] show that the multiple-description code can dramatically improve the stability and performance of Kalman filters over packetdropping links while efficiently using the communication bandwidth. Although the work of [6] and [7] provide great insights into the state estimation problem with unreliable communication links, their results are restricted to linear dynamics with Gaussian noises. State estimation with nonlinear dynamics and non-Gaussian noises over a packet-dropping communication network has not been widely investigated yet.

Part of the difficulty with nonlinear dynamics and nonGaussian noises is that theoretical guarantees are often hard to come by. Many strategies exist for online nonlinear estimation and we focus on two such strategies in this paper: the extended Kalman filter (EKF) and moving horizon estimation (MHE). Due to the ease of implementation and a widespread range of applications, many theoretical properties of the EKF have been explored: [8] shows that, when either the initial estimate is close enough to the true value or the nonlinearity of the system is small enough, the EKF converges locally. Sufficient conditions that guarantee stochastic stability are derived in [9]. In [10], the authors linked the convergence behavior of the EKF to the derivative of the nonlinear dynamics. However, as noted by some researchers, the existing convergence conditions are generally too conservative so that they are mainly of the theoretical interest.

MHE is an approach for online state estimation problem with nonlinear dynamics, constrained variables, and nonquadratic costs. The computation complexity is bounded by using a finite-size moving horizon window. As new measurements become available, old measurements are discarded, and the state estimation problem is resolved inside the horizon window. It has been shown in [11] that this approach can be used in some applications where the EKF is not appropriate. If the arrival cost is known exactly, then MHE provides the optimal Bayesian estimate. However, the arrival cost is difficult to compute in practice, and thus must be approximated. In such case, there are no known optimality guarantees. For a more in-depth discussion of MHE, we refer readers to [11].

Despite the lack of general performance guarantees, non- 
linear systems arise often in practice. In today's networked environment, it is important to understand the performance of nonlinear estimation schemes. We investigate the performance of both EKF and MHE for estimating the state of a nonlinear dynamical system with white Gaussian disturbance and observation noise over a packet-dropping network. Comparing with the result on the EKF, which is a straight extension from the linear case, using MHE to deal with packet drops is a brand-new idea. We use a large-scale nonlinear programming software package, SNOPT, to solve the numerical optimization problem that arises in MHE.

The remainder of this paper is organized as follows. In Section II, the formulation of an extended Kalman filter with an i.i.d. Bernoulli packet-dropping network is presented. We give a sufficient condition on the boundedness of the expected EKF error covariance. In Section III, we discuss the method of moving horizon estimation with the details of SNOPT programming, where a new estimation strategy for packet-dropping is proposed. Examples and simulation results are provided in Section IV and we conclude with remarks on future research directions in Section V.

\section{EXTENDED KALMAN FILTER WITH OBSERVATION LOSS}

For simplicity, we consider a nonlinear discrete-time dynamical system without control inputs

$$
\begin{aligned}
x_{k+1} & =f\left(x_{k}\right)+w_{k} \\
y_{k} & =h\left(x_{k}\right)+v_{k}
\end{aligned}
$$

where $x_{k} \in \mathbb{R}^{n}$ is the state, $y_{k} \in \mathbb{R}^{m}$ is the output, $w_{k}$ and $v_{k}$ are independent zero-mean, white Gaussian noise processes with covariances $Q>0$ and $R>0$, respectively. We assume that $f(\cdot)$ and $h(\cdot)$ are at least twice differentiable.

\section{A. EKF without packet-dropping}

The extended Kalman filter can be represented in two parts: the time update

$$
\left\{\begin{array}{l}
\hat{x}_{k+1}^{-}=f\left(\hat{x}_{k}\right) \\
P_{k+1}^{-}=A_{k} P_{k} A_{k}^{T}+Q
\end{array}\right.
$$

and the measurement update

$$
\left\{\begin{array}{l}
\hat{x}_{k}=\hat{x}_{k}^{-}+K_{k}\left(y_{k}-h\left(\hat{x}_{k}^{-}\right)\right) \\
P_{k}=\left(I-K_{k} C_{k}\right) P_{k}^{-}
\end{array}\right.
$$

where

$$
\begin{aligned}
A_{k} & =\frac{\partial f}{\partial x}\left(\hat{x}_{k}\right) \\
C_{k} & =\frac{\partial h}{\partial x}\left(\hat{x}_{k}^{-}\right) \\
K_{k} & =P_{k}^{-} C_{k}^{T}\left(C_{k} P_{k}^{-} C_{k}^{T}+R\right)^{-1} .
\end{aligned}
$$

Let $g_{k}(\cdot)$ denote the Riccati update for the error covariance

$$
\begin{aligned}
g_{k}(X)= & A_{k} X A_{k}^{T}+Q \\
& -A_{k} X C_{k}^{T}\left(C_{k} X C_{k}^{T}+R\right)^{-1} C_{k} X^{T} A_{k}^{T} .
\end{aligned}
$$

Since $A_{k}$ and $C_{k}$ are time-variant and they depend on the estimate at each step, it is difficult to give general conditions on uniform boundness of the error covariance. Let us define the map $H: \mathbb{R}^{n} \rightarrow \mathbb{R}^{m \times n}$ as

$$
H(x)=\left[h(x) ; h(f(x)) ; \cdots ; h\left(f^{n-1}(x)\right)\right]
$$

where

$$
f^{n-1}(x)=\underbrace{f(f(\cdots f(\cdot)))}_{n-1}
$$

denotes function composition.

A nonlinear system is said to satisfy the observability rank condition if the rank of

$$
\frac{\partial H}{\partial x}\left(x_{0}\right)=\left[\begin{array}{l}
\frac{\partial h}{\partial x}\left(x_{0}\right) \\
\frac{\partial h}{\partial x}\left(x_{1}\right) \frac{\partial f}{\partial x}\left(x_{0}\right) \\
\vdots \\
\frac{\partial h}{\partial x}\left(x_{n-1}\right) \frac{\partial f}{\partial x}\left(x_{n-2}\right) \cdots \frac{\partial f}{\partial x}\left(x_{0}\right)
\end{array}\right]
$$

equals $n$ for any $x_{0} \in \mathbb{R}^{n}$. According to [8], [9], if system (1) satisfies the observability rank condition, the uniformly bounded error covariance of the associated EKF is a sufficient condition for the estimation error $e_{k}=x_{k}-\hat{x}_{k}$ of the EKF to be exponentially bounded, as long as either the initial guess is close enough to the true value or the function $f(\cdot)$ is only weakly nonlinear. For a precise statement of the sufficient condition, we refer readers to Theorem 3.1 of [9] or Theorem 5.2 of [8]. The exact statement is omitted to avoid excess notations.

\section{B. EKF with packet-dropping}

We model the packet-dropping process as an i.i.d. Bernoulli random process. A sequence of Bernoulli random variables $\gamma_{k}$ is used to indicate whether a packet is successfully transmitted at time $k$. More precisely, if $\gamma_{k}=1$ then the packet goes through the communication network; otherwise, $\gamma_{k}=0$ and the packet is dropped. This random process is characterized by a single parameter $\lambda$ :

$$
\gamma_{k}= \begin{cases}1 & \text { with probability } \lambda \\ 0 & \text { with probability } 1-\lambda\end{cases}
$$

When a packet is lost, we proceed naturally with the timeupdate step. In the case of linear systems and Gaussian noise, this has been shown to be optimal in [6]. The Riccati update for the EKF is

$$
g_{k}^{0}(X)=A_{k} X A_{k}^{T}+Q
$$

when $\gamma_{k}=0$, and

$$
\begin{aligned}
g_{k}^{1}(X)= & A_{k} X A_{k}^{T}+Q \\
& -A_{k} X C_{k}^{T}\left(C_{k} X C_{k}^{T}+R\right)^{-1} C_{k} X^{T} A_{k}^{T}
\end{aligned}
$$

when $\gamma_{k}=1$. Thus, the error covariance recurrence of the EKF is stochastic and we have the following theorem.

Theorem 2.1: Consider the nonlinear system (1) with the following properties

1) The system satisfies the observability rank condition in Equation (7);

2) The first-order derivative $\frac{\partial f}{\partial x}$ is invertible for any $x \in$ $\mathbb{R}^{n}$ 
3) There exists a detectible pair $(A, C)$ such that

$$
A \geq\left.\frac{\partial f}{\partial x}\right|_{x=x_{0}} \text { and } C^{T} R^{-1} C \leq\left.\frac{\partial h^{T}}{\partial x} R^{-1} \frac{\partial h}{\partial x}\right|_{x=x_{0}}
$$

for all $x_{0} \in \mathbb{R}^{n}$.

Then, the expected error covariance $E\left[P_{k}^{-}\right]$is uniformly bounded if

$$
\lambda>1-1 / \rho(A)^{2}
$$

where $\rho(A)$ is the spectral radius of $A$.

Proof: The first two properties guarantee that $P_{k}^{-}$is uniformly bounded without packet drops [8], [9]. In order to show $E\left[P_{k}^{-}\right]>0$ is uniformly bounded with packet drops, we need to find an upper bound. Let

$$
\tilde{g}^{0}(X)=A X A^{T}+Q
$$

and

$$
\begin{aligned}
\tilde{g}^{1}(X)= & A X A^{T}+Q \\
& -A X C^{T}\left(C X C^{T}+R\right)^{-1} C X^{T} A^{T} .
\end{aligned}
$$

It is true that

$$
\left\{\begin{array}{l}
g_{k}^{0}(X) \leq \tilde{g}^{0}(X) \\
g_{k}^{1}(X) \leq \tilde{g}^{1}(X)
\end{array}\right.
$$

for any $k$. The first inequality is obvious from the definition of $A$. For the second inequality, note that the update for error covariance in the EKF can be re-written using the matrix inversion lemma.

$$
\left\{\begin{array}{l}
P_{k+1}^{-}=A_{k} P_{k} A_{k}^{T}+Q \\
P_{k}^{-1}=\left(P_{k}^{-}\right)^{-1}+C_{k}^{T} R^{-1} C_{k} .
\end{array}\right.
$$

Thus, with the same initial conditions, the error covariance $P_{k}^{-}$is bounded by the error covariance $\tilde{P}_{k}^{-}$. Here, $\tilde{P}_{k}^{-}$ corresponds to the Kalman filter error covariance for the linear system

$$
\left\{\begin{aligned}
x_{k+1} & =A x_{k}+w_{k} \\
y_{k} & =C x_{k}+v_{k} .
\end{aligned}\right.
$$

So we have

$$
E\left[P_{k}^{-}\right] \leq E\left[\tilde{P}_{k}^{-}\right] .
$$

According to [6], [7], the expected value $E\left[\tilde{P}_{k}^{-}\right]$evolves according to the modified ARE

$$
\begin{aligned}
g_{\lambda}(X)= & A X A^{T}+Q \\
& -\lambda A X C^{T}\left(C X C^{T}+R\right)^{-1} C X^{T} A^{T} .
\end{aligned}
$$

And it has been shown in [6], [7] that $E\left[\tilde{P}_{k}^{-}\right]$converges to a unique positive definite matrix, i.e. uniformly bounded, as $k \rightarrow \infty$ if the packet-dropping rate $1-\lambda$ satisfies

$$
1-\lambda<1 / \rho(A)^{2} .
$$

This theorem states a sufficient condition on the uniform boundedness of the error covariance of the EKF with packetdropping. It indicates that the EKF exhibits a similar phase transition as the Kalman filter with respect to packet drops. However, we have the following comments on this result:
- First, this condition is conservative since the behavior of the EKF is bounded by a Kalman filter of an approximate linear system. This conservativeness is verified in Section IV by simulation results.

- It is well known that the Riccati update is only a first-order approximation to the true error covariance. In other words, the uniform boundedness of $P_{k}^{-}$does not necessarily indicate the boundedness of $e_{k}$. Other conditions on the linearity of $f(\cdot)$ and $h(\cdot)$ as well as the precision of the initial guess must be considered. For the EKF with packet-dropping, Theorem 2.1 can only be used to judge the boundedness of $E\left[P_{k}^{-}\right]$. The behavior of $E\left[e_{k}\right]$ is still under investigation.

\section{MOVING HORIZON ESTIMATION WITH PACKET-DROPPING}

Other than EKF, moving horizon estimation (MHE) is another method to estimate a nonlinear system (1), which is formulated as an optimization problem to handle constraints explicitly. For simplicity, we assume that $h(x)=x$. The optimization problem solves

$$
\min _{x_{0},\left\{w_{k}\right\}_{k=0}^{T-1}} \sum_{k=0}^{T-1}\left\|w_{k}\right\|_{Q^{-1}}^{2}+\left\|v_{k}\right\|_{R^{-1}}^{2}+\left\|x_{0}-\hat{x}_{0}\right\|_{\Pi^{-1}}^{2}
$$

at time $T$. As $T$ increases, more observation data are taken into account and the optimization increases in size. To limit the amount of computation, MHE considers a finite-size horizon window. Fig. 2 illustrates this concept. When the time step increases by one, the horizon window moves one step to the right by including one new observation data and discarding the oldest one. More precisely, MHE solves the following optimization at each time $T$

$$
\begin{array}{ll}
\min _{x_{T-N+1},\left\{w_{k}\right\}_{T-N}^{T-1}} & \sum_{k=T-N}^{T-1}\left\|w_{k}\right\|_{Q^{-1}}^{2}+\left\|v_{k}\right\|_{R^{-1}}^{2} \\
& +\mathcal{Z}_{T-N+1}\left(x_{T-N+1}\right)
\end{array}
$$

where $N$ is the horizon window size and $\mathcal{Z}_{T-N+1}\left(x_{T-N+1}\right)$ is called the arrival cost, which summarizes the past information up to time $T-N+1$. For general nonlinear systems with the form (1), it is difficult to determine the true arrival cost. As often done in practice, we run an EKF with MHE at the same time and use the weighted deviation from the result of the EKF as the arrival cost. In symbols,

$$
\mathcal{Z}_{T-N-1}\left(x_{T-N-1}\right)=\left\|x_{T-N-1}-\hat{x}_{T-N-1}\right\|^{2} \cdot \Pi_{T-N-1}^{-1}
$$

where $\Pi_{T-N-1}$ is the error covariance of the EKF.

\section{A. Formulation of MHE with SNOPT}

A MHE scheme needs to solve an optimization problem at each step. We use SNOPT [12], a general-purpose software package, as our numerical solver. In the cost function (16), there are $2 N+2$ variables total and they are $\left\{x_{T-N+1}, w_{T-N+1}, \cdots, w_{T-1}, v_{T-N+1}, \cdots, v_{T}\right\}$. Our goal is to solve (16) subject to the following $N-1$ nonlinear 


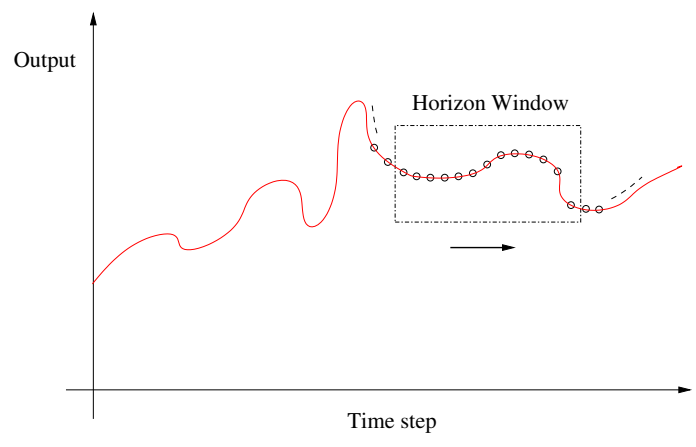

Fig. 2. Diagram of MHE with no packet-dropping.

constraints:

$$
\left\{\begin{array}{ccc}
f\left(x_{T-N+1}\right)+v_{T-N+2}+w_{T-N+1}-y_{T-N+2} & = & 0 \\
f\left(y_{T-N+2}-v_{T-N+2}\right)+v_{T-N+3} & & \\
+w_{T-N+2}-y_{T-N+3} & = & 0 \\
f\left(y_{T-1}-v_{T-1}\right)+v_{T}+w_{T-1}-y_{T} & \vdots & 0
\end{array}\right.
$$

and one linear constraint

$$
x_{T-N+1}+v_{T-N+1}-y_{T-N+1}=0 .
$$

These equality constraints arise from the system dynamics. Additional inequality constraints on variables can be introduced to model, for example, bounded noise.

To compute the Jacobian matrix of those constraints, we order those variables as follows

$$
\{\underbrace{x_{T-N+1}, v_{T-N+2}, \cdots, v_{T}}_{N \text { nonlinear Jacobian variables }}, \underbrace{w_{T-N+1}, \cdots, w_{T-1}, v_{T-N+1}}_{\text {N linear Jacobian variables }}\}
$$

to yield the sparse Jacobian matrix as

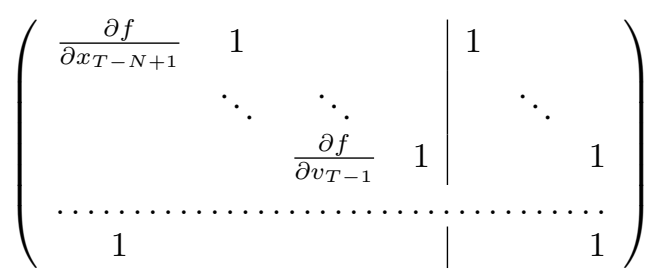

where the left-upper $(N-1) \times(N-1)$ sub-matrix is the nonlinear Jacobian matrix and the last row corresponds to the linear constraint.

After solving this optimization problem, the estimation of $x_{T}$ can be calculated by

$$
\begin{aligned}
x_{T-N+2} & =f\left(x_{T-N+1}\right)+w_{T-N+1} \\
x_{T-N+3} & =f\left(x_{T-N+2}\right)+w_{T-N+2} \\
& \downarrow \\
x_{T} & =f\left(x_{T-1}\right)+w_{T-1} .
\end{aligned}
$$

\section{B. Estimation strategy for packet-dropping}

When a packet is dropped in the communication network, the estimator has to predict the state value at that time step. For the EKF, it is natural to proceed with the time update

\begin{tabular}{|c|c|c|c|c|c|c|c|}
\hline \multicolumn{2}{|c|}{ Time index } & $i_{1}$ & $i_{2}$ & . & & $i_{N-1}$ & $i_{N}$ \\
\hline \multicolumn{2}{|c|}{ Packet value } & $y_{i_{1}}$ & $y_{i_{2}}$ & $\cdot$ & & $y_{i_{N-1}}$ & $y_{i_{N}}$ \\
\hline $\begin{array}{c}\text { Time } \\
\text { intervals }\end{array}$ & \multicolumn{2}{|c|}{$\begin{array}{c}m_{1}= \\
i_{2}-i_{1}\end{array}$} & \multicolumn{2}{|c|}{$\begin{array}{c}m_{2}= \\
i_{3}-i_{2}\end{array}$} & $\cdots$ & \multicolumn{2}{|c|}{$\begin{array}{c}m_{N-1}= \\
i_{N}-i_{N-1}\end{array}$} \\
\hline
\end{tabular}
step until a packet is successfully received. For MHE, we propose the following strategy to handle packet loss.

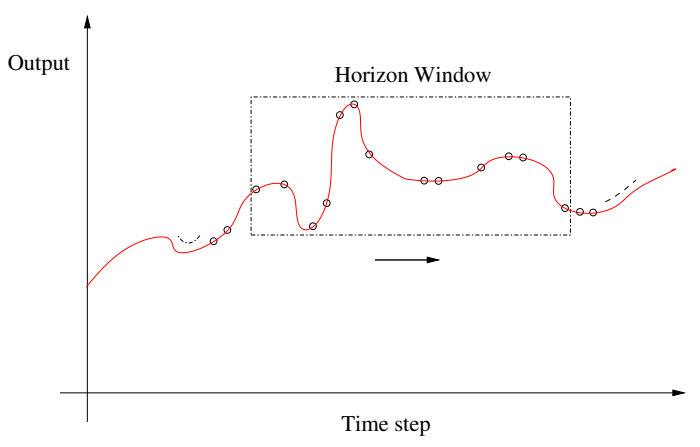

Fig. 3. Diagram of MHE with packet-dropping.

TABLE I

RECEIVED PACKET HISTORY AND TIME INTERVALS

If the packet is dropped at time $k$, i.e. the estimator does not receive $y_{k}$. We estimate the state at time $k$ as

$$
\hat{x}_{k}=f\left(\hat{x}_{k-1}\right)
$$

This one-step propagation method is used whenever the packet is dropped. If consecutive packets are dropped, we perform this time update multiple times.

If the packet is received at time $k$, the estimator uses the latest $N$ received observation data as nonlinear constraints. Because multiple packets may be dropped in succession, the time indices of the last $N$ received packets may not be consecutive. Fig. 3 shows this strategy. The width of the horizon may vary at each step, but the number of successfully received observation packets inside the window is constant. When a new packet arrives, the horizon window moves to the right by discarding the oldest observation data. Based on this strategy, the estimator only needs to store the latest $N$ received packets at any time. Table I shows an example of the memory and time intervals.

Due to the packet loss, we must use a different cost function in the optimization as

$$
\min _{x_{i_{1}},\left\{w_{k}\right\}_{i_{1}}^{i_{N}}} \sum_{k=i_{1}}^{i_{N}}\left\|w_{k}\right\|_{Q^{-1}}^{2}+\left\|v_{k}\right\|_{R^{-1}}^{2}+\left\|x_{i_{1}}-\hat{x}_{i_{1}}\right\|^{2} \cdot \Pi_{i_{1}}^{-1} .
$$

The arrival cost is again based on the output of an EKF at time $i_{1}$. The new optimization variables are

$$
\{\underbrace{x_{i_{1}}, v_{i_{2}}, \cdots, v_{i_{N}}}_{N \text { nonlinear Jacobian variables }}, \underbrace{w_{i_{1}-1}, \cdots, w_{i_{N-1}}, v_{i_{1}}}_{N \text { linear Jacobian variables }}\} .
$$


The nonlinear constraints are

$$
\left\{\begin{array}{cc}
f^{m_{1}}\left(x_{i_{1}}\right)+v_{i_{2}}+w_{i_{1}}-y_{i_{2}} & =0 \\
f^{m_{2}\left(y_{i_{2}}-v_{i_{2}}\right)+v_{i_{3}}+w_{i_{2}}-y_{i_{3}}} & =0 \\
f^{m_{N-1}}\left(y_{i_{N-1}}-v_{i_{N-1}}\right)+v_{i_{N}}+w_{i_{N-1}}-y_{i_{N}} & =0
\end{array}\right.
$$

and the linear constraint

$$
x_{i_{1}}+v_{i_{1}}-y_{i_{1}}=0 .
$$

The difference between constraints in (18) and (24) is that the nonlinear function $f(\cdot)$ is replaced by compositions $f^{m}(\cdot)$. While the Jacobian matrix has the same form as in Equation (20).

\section{EXAMPLES AND SIMULATION RESULTS}

In this section, we apply the aforementioned estimation strategies to two scalar nonlinear systems as examples.

\section{A. Example: a stable nonlinear system}

Let us first consider the system

$$
\left\{\begin{aligned}
x_{k+1} & =x_{k}-0.001 \cdot x_{k}\left(x_{k}+2\right)\left(x_{k}-5\right)+w_{k} \\
y_{k} & =x_{k}+v_{k},
\end{aligned}\right.
$$

where $w_{k}$ and $v_{k}$ are zero-mean white Gaussian noise processes with covariances $Q=0.01$ and $R=6$, respectively. This system satisfies the observability rank condition and has three equilibrium points $\{0,-2,5\}$, where -2 and 5 are stable equilibrium points and 0 is unstable. As a comparison, we first consider the case with no packet-drops. Fig. 4 shows the performance of EKF and MHE with window size 70. It is apparent that with Gaussian noise and stable dynamics, the EKF is almost as good as MHE. Fig. 5 shows the horizon window of MHE. The red interval represents the estimated state values inside the horizon window based on the results of the numerical solver, SNOPT. The green curve shows the actual states, the cyan dots are the noisy observations, and the blue is the estimated trajectory according to MHE. We ran simulations on a desktop computer with an Intel(R) Xeon(R) CPU at $2.66 \mathrm{GHz}$ and $1 \mathrm{~GB}$ of RAM. The update rate of MHE about $1 \mathrm{~Hz}$.

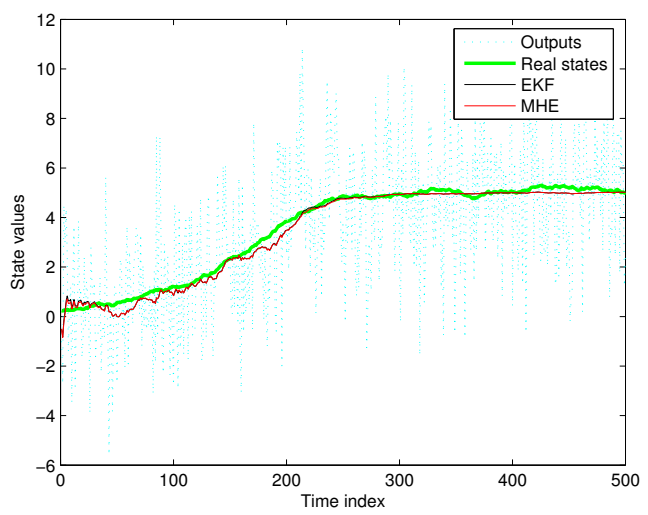

Fig. 4. EKF and MHE with no packet-dropping.

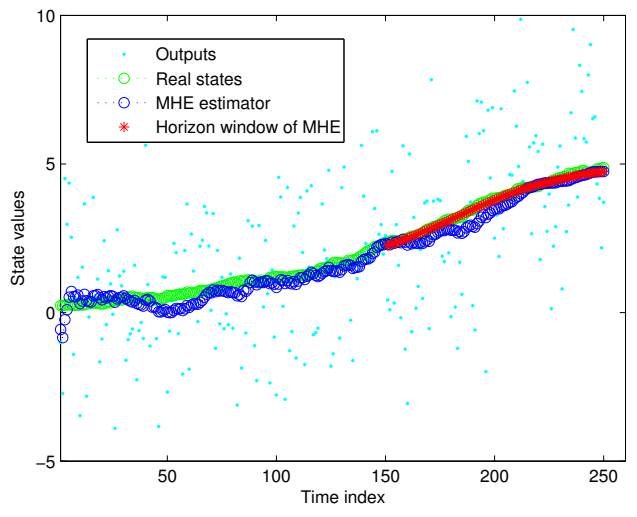

Fig. 5. Horizon window of MHE with no packet-dropping.

Fig. 6 shows the simulation result of the EKF under various packet-dropping conditions. Large packet drop rates degrade estimator performance regardless which approach is used. We get the similar figure for MHE as well. Since the dynamics are stable, both EKF and MHE eventually converge to the stable state. The output of estimator breaks into discontinuous pieces at high packet-dropping rate. Each piece corresponds to an interval of prediction due to continuous packet drops. When a packet successfully received, the estimator updates its output and the estimated trajectory jumps. Fig. 7 shows a typical horizon window of MHE. The red cross represents the estimated state values inside the horizon window. The green curve shows the true states, the cyan dots denote the received noisy outputs which is quite sparse due to the high loss rate (40\%), and the black is the estimated trajectory. Since the system (26) is rather tame, we can attribute the comparable performance to the fact that the arrival cost of MHE is determined by an EKF.

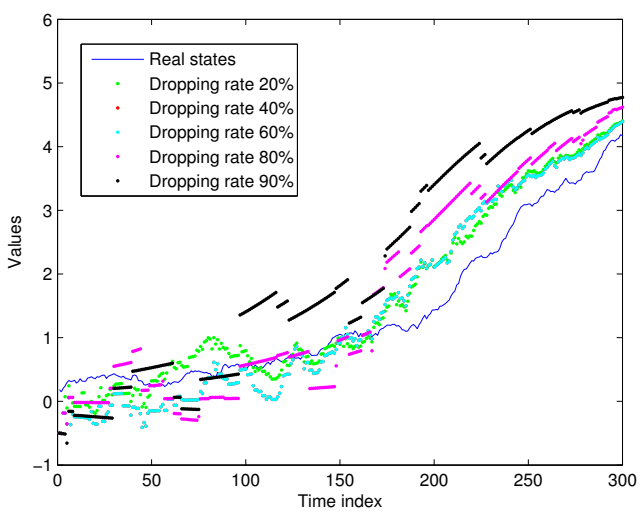

Fig. 6. EKF with packet-dropping.

\section{B. Example: an unstable nonlinear system}

The second system that we consider is

$$
\left\{\begin{aligned}
x_{k+1} & =1.1 \cdot x_{k}+0.2 \cdot \sin \left(x_{k}\right)+w_{k} \\
y_{k} & =x_{k}+v_{k}
\end{aligned}\right.
$$




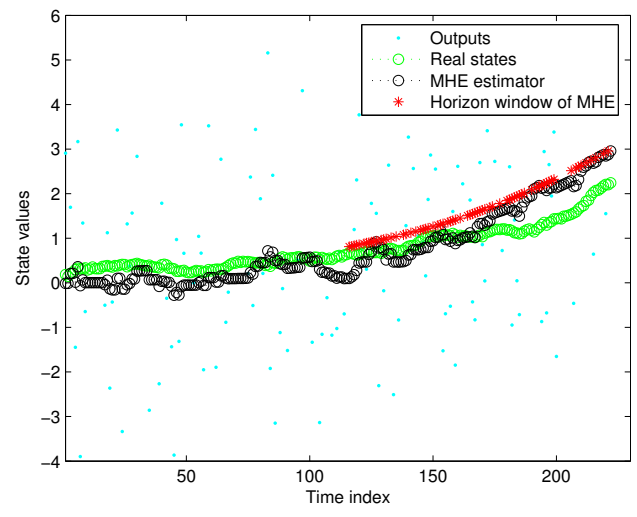

Fig. 7. Horizon window of MHE with $20 \%$ packet-dropping.

where $w_{k}$ and $v_{k}$ are zero-mean Gaussian white noise processes with covariances $Q=0.01$ and $R=6$, respectively. This system only has one equilibrium point at 0 and it is unstable.

Obviously, the nonlinear system (27) satisfies the observability rank condition since $y_{k}=x_{k}+v_{k}$. The derivative of $f(\cdot)$ is bounded by

$$
0.9 \leq \frac{\partial f}{\partial x} \leq 1.3 .
$$

According to Theorem 2.1, the sufficient condition for uniform boundedness of the expected error covariance is $\lambda>0.41$, i.e., the packet-dropping rate should be below $59 \%$. Fig. 8 shows the simulation result for the EKF where the average error covariance dose not start to diverge until the packet-dropping rate is over $80 \%$. This is a good example of the conservativeness of Theorem 2.1.

For the estimated trajectories of EKF and MHE, they are similar to Fig. 6. We omit them due to space limitations.

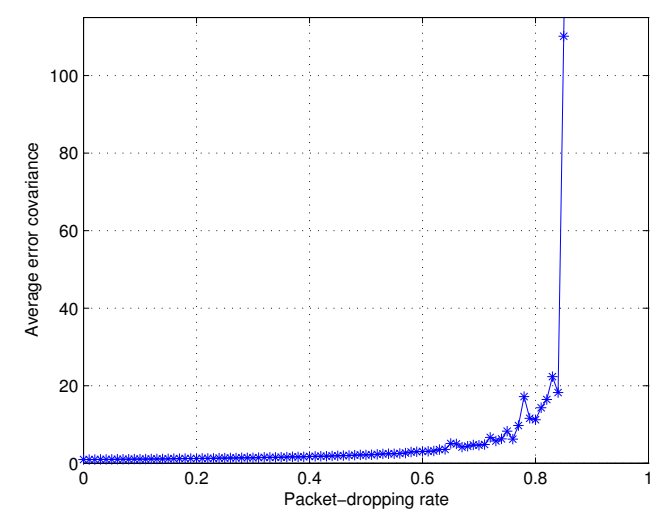

Fig. 8. Average error covariance of the EKF with different packet-dropping rates.

\section{CONCLUSIONS AND FUTURE WORK}

In this paper, we investigated the state estimation problem for nonlinear dynamical systems with packet drops. For the EKF, we state a sufficient condition on the uniform boundness of the expected error covariance. Even though this condition is conservative, it indicates the existence of a phase transition in an EKF, which is similar to the linear case. For MHE, we introduced an estimation strategy to deal with the intermittent data. When packet-dropping occurs, the estimator conducts prediction. When a packet is received, the packet history stored in the estimator is updated and the nonlinear constraints in MHE are reformulated correspondingly. Simulation results are presented for two scalar nonlinear dynamics for both EKF and MHE. The simulation results verify the expected behaviors of these two estimators.

The future work includes a few issues. First of all, we would like to study the case with non-Gaussian noise so that we can compare the statistical behaviors of error covariance of EKF and MHE; Second, it is interesting to investigate the behavior of expected error $e_{k}$ of the EKF with packet drops. Lastly, we would like to investigate whether multiple description codes can improve the performance.

\section{REFERENCES}

[1] P. Antsaklis and J. Baillieul, Eds., IEEE Transactions on Automatic Control, Sep. 2004, vol. 49, no. 9, special Issue on Networked Control Systems.

[2] N. Nahi, "Optimal recursive estimation with uncertain observation," IEEE Transactions on Information Theory, vol. 15, no. 4, pp. 457462, Apr. 1969.

[3] M. Hadidi and S. Schwartz, "Linear recursive state estimators under uncertain observations," IEEE Transactions on Information Theory, vol. 24, no. 6, pp. 944-948, Dec. 1979.

[4] J. Nilsson, B. Bernhardsson, and B. Wittenmark, "Stochastic analysis and control of real-time systems with random time delays," Automatica, vol. 34, no. 1, pp. 57-64, Jan. 1998.

[5] O. Costa, "Stationary filter for linear minimum mean square error estimator of discrete-time Markovian jump systems," IEEE Transactions on Automatic Control, vol. 47, no. 8, pp. 1351-1356, Aug. 2002.

[6] B. Sinopoli, L. Schenato, M. Franceschetti, K. Poolla, M. I. Jordan, and S. S. Sastry, "Kalman filtering with intermittent observations," IEEE Transactions on Automatic Control, vol. 49, no. 9, pp. 14531464, Sep. 2004.

[7] Z. Jin, V. Gupta, and R. M. Murray, "State estimation over packet dropping networks using multiple description coding," Automatica, vol. 42, no. 9, pp. 1441-1452, Sep. 2006.

[8] Y. Song and J. W. Grizzle, "The extended kalman filter as a local asymptotic observer for discrete-time nonlinear systems," Journal of Mathematical Systems, Estimation, and Control, vol. 5, no. 1, pp. 5978, 1995.

[9] K. Reif, S. Gunther, E. Yaz, and R. Unbehauen, "Stochastic stability of the discrete-time extended kalman filter," IEEE Transactions on Automatic Control, vol. 44, no. 4, pp. 714-728, Apr. 1999.

[10] T. Zhai, H. Ruan, and E. E. Yaz, "Performance evaluation of extended kalman filter based state estimation for first order nonlinear dynamic systems," in Proceedings of the 42nd IEEE Conference on Decision and Control, vol. 2, 2003, pp. 1386-1391.

[11] C. Rao, J. Rawlings, and D. Mayne, "Constrained state estimation for nonlinear discrete-time systems: Stability and moving horizon approximations," IEEE Transactions on Automatic Control, vol. 48, no. 2, pp. 246-258, 2003.

[12] P. E. Gill, W. Murray, and M. A. Saunders, User's gude for SNOPT 5.3: A fortran package for large-scale nonlinear programming, Systems Optimization Laboratory, Stanford University, Feb. 1999. [Online]. Available: http://citeseer.ist.psu.edu/gill99users.html 